UT-03-03

\title{
Lattice chiral symmetry, CP-violation and Majorana fermions
}

\author{
Kazuo Fujikawa \\ Department of Physics, University of Tokyo \\ Bunkyo-ku,Tokyo 113, Japan
}

\begin{abstract}
A brief summary of lattice fermions defined by the general Ginsparg-Wilson algebra is first given. It is then shown that those general class of fermion operators have a conflict with $\mathrm{CP}$ invariance in chiral gauge theory and with the definition of Majorana fermions in the presence of chiral-symmetric Yukawa couplings. The same conclusion holds for the domain-wall fermion also.
\end{abstract}

\section{Introduction}

The recent developments in the analysis of chiral symmetry in lattice theory is based on the so-called Ginsparg-Wilson relation[1]. Neuberger found an explicit construction of lattice fermion operator (the overlap operator)[2], which was later shown to satisfy the Ginsparg-Wilson relation. This operator was then shown to satisfy an index theorem on the lattice under certain conditions[3]. This index relation was used by Lüscher to derive the chiral anomaly as a Jacobian factor[4] just as in continuum theory[5]. These topological properties were later examined in further detail[6]. See Refs.[7] for reviews of these developments.

To be more specific, we here work on the Ginsparg-Wilson relation defined by[8]

$$
\gamma_{5}\left(\gamma_{5} D\right)+\left(\gamma_{5} D\right) \gamma_{5}=2 a^{2 k+1}\left(\gamma_{5} D\right)^{2 k+2}
$$

where $D$ is the lattice Dirac operator and $k$ stands for a non-negative integer; $k=0$ corresponds to the ordinary Ginsparg-Wilson relation.

We deal with a hermitian lattice operator

$$
H=a \gamma_{5} D=H^{\dagger}=a D^{\dagger} \gamma_{5}
$$

and the above algebra is written as

$$
\gamma_{5} H+H \gamma_{5}=2 H^{2 k+2}
$$

where $k=0$ corresponds to conventional Ginsparg-Wilson relation. We also assume that the operator $H$ is local in the sense that it is analytic in the entire Brillouin zone and free 
of species doublers, which is in fact shown for the free operator without gauge field[9]. One can then confirm the relation

$$
\gamma_{5} H^{2}=H^{2} \gamma_{5}
$$

The defining algebra is written in various ways such as

$$
\begin{aligned}
& \Gamma_{5} H+H \Gamma_{5}=0, \\
& \gamma_{5} H+H \hat{\gamma}_{5}=0, \\
& \hat{\gamma}_{5}^{2}=1
\end{aligned}
$$

where

$$
\begin{aligned}
& \Gamma_{5}=\gamma_{5}-H^{2 k+1}, \\
& \hat{\gamma}_{5}=\gamma_{5}-2 H^{2 k+1} .
\end{aligned}
$$

We can also show

$$
\left(\gamma_{5} \Gamma_{5}\right) \hat{\gamma}_{5}=\gamma_{5}\left(\gamma_{5} \Gamma_{5}\right)
$$

We now examine the action defined by

$$
S=\int d^{4} x \bar{\psi} D \psi \equiv \sum_{x, y} \bar{\psi}(x) D(x, y) \psi(y)
$$

which is invariant under

$$
\delta \psi=i \epsilon \hat{\gamma}_{5} \psi, \quad \delta \bar{\psi}=\bar{\psi} i \epsilon \gamma_{5}
$$

If one considers the field re-definition

$$
\psi^{\prime}=\gamma_{5} \Gamma_{5} \psi, \quad \bar{\psi}^{\prime}=\bar{\psi}
$$

the above action is written as

$$
S=\int d^{4} x \bar{\psi}^{\prime} D \frac{1}{\gamma_{5} \Gamma_{5}} \psi^{\prime}
$$

which is invariant under naive chiral transformation

$$
\begin{aligned}
& \delta \psi^{\prime}=i \epsilon \gamma_{5} \psi^{\prime}, \\
& \delta \bar{\psi}^{\prime}=\bar{\psi}^{\prime} i \epsilon \gamma_{5} .
\end{aligned}
$$

This chiral symmetry implies the relation

$$
\left\{\gamma_{5}, D \frac{1}{\gamma_{5} \Gamma_{5}}\right\}=0
$$


This naive chiral symmetry of the species doubler-free operator suggests the non-analytic behavior of the factor $1 /\left(\gamma_{5} \Gamma_{5}\right)$ in the Brillouin zone. In fact, one can confirm that $[9,10]$

$$
\Gamma^{2} \equiv \Gamma_{5}^{2}=1-H^{4 k+2}=0
$$

has solutions at the momentum variables corresponding to would-be species doublers, in the case of free operator without the gauge field, and also in the presence of topologically non-trivial gauge field. See also Ref.[11].

We next recall the charge conjugation properties

$$
\begin{aligned}
& C \gamma^{\mu} C^{-1}=-\left(\gamma^{\mu}\right)^{T}, \quad C \gamma_{5} C^{-1}=\gamma_{5}^{T}, \\
& C^{\dagger} C=1, \quad C^{T}=-C .
\end{aligned}
$$

We then have ${ }^{1}$

$$
\begin{aligned}
& W D\left(U^{\mathrm{CP}}\right) W^{-1}=D(U)^{T}, \quad W \gamma_{5} \Gamma_{5}\left(U^{\mathrm{CP}}\right) W^{-1}=\left[\gamma_{5} \Gamma_{5}(U)\right]^{T}, \\
& W H\left(U^{\mathrm{CP}}\right) W^{-1}=-\left[\gamma_{5} H(U) \gamma_{5}\right]^{T}, \quad W H^{2}\left(U^{\mathrm{CP}}\right) W^{-1}=\left[H^{2}(U)\right]^{T}, \\
& W \Gamma_{5}\left(U^{\mathrm{CP}}\right) W^{-1}=-\left[\gamma_{5} \Gamma_{5}(U) \gamma_{5}\right]^{T}, \\
& W\left(\Gamma_{5} / \Gamma\right)\left(U^{\mathrm{CP}}\right) W^{-1}=-\left[\left(\gamma_{5} \Gamma_{5} \gamma_{5} / \Gamma\right)(U)\right]^{T}
\end{aligned}
$$

where

$$
\Gamma=\sqrt{\Gamma_{5}^{2}}=\sqrt{\left(\gamma_{5} \Gamma_{5} \gamma_{5}\right)^{2}}=\sqrt{1-H^{4 k+2}} .
$$

Here we imposed the relation $W D\left(U^{\mathrm{CP}}\right) W^{-1}=D(U)^{T}$ or $[C D(U)]^{T}=-C D\left(U^{\mathrm{C}}\right)$ which is consistent with the defining Ginsparg-Wilson relation.

We also have the property

$$
W \hat{\gamma}_{5}\left(U^{\mathrm{CP}}\right) W^{-1}=-\left[\gamma_{5} \hat{\gamma}_{5}(U) \gamma_{5}\right]^{T} .
$$

\section{CP symmetry in lattice chiral gauge theory}

We now examine the CP symmetry in chiral gauge theory

$$
\mathcal{L}_{L}=\bar{\psi}_{L} D \psi_{L}
$$

where we defined the (general) projection operators

$$
\begin{aligned}
& D=\bar{P}_{L} D P_{L}+\bar{P}_{R} D P_{R}, \\
& \psi_{L, R}=P_{L, R} \psi, \quad \bar{\psi}_{L, R}=\bar{\psi} \bar{P}_{L, R} .
\end{aligned}
$$

\footnotetext{
${ }^{1}$ We define the CP operation by $W=C \gamma_{0}=\gamma_{2}$ with hermitian $\gamma_{2}$ and the CP transformed gauge field by $U^{\mathrm{CP}}$, and then $W D\left(U^{\mathrm{CP}}\right) W^{-1}=D(U)^{T}$. If the parity is realized in the standard way, we have $C D\left(U^{\mathrm{C}}\right) C^{-1}=D(U)^{T}$.
} 
It was pointed out by Hasenfratz[12] that the conventional Ginsparg-Wilson operator when applied to chiral gauge theory has a difficulty with CP symmetry. We would like to examine this issue in more detail. Under the standard CP transformation ${ }^{2}$

$$
\begin{aligned}
& \bar{\psi} \rightarrow \psi^{T} W, \\
& \psi \rightarrow-W^{-1} \bar{\psi}^{T}
\end{aligned}
$$

the chiral action is invariant only if

$$
W P_{L} W^{-1}=\bar{P}_{L}^{T}, \quad W \bar{P}_{L} W^{-1}=P_{L}^{T} .
$$

It was shown in Ref. [13] that the unique solution for this condition in the framework of the Ginsparg-Wilson operators is given by

$$
\begin{array}{r}
P_{L, R}=\frac{1}{2}\left(1 \mp \Gamma_{5} / \Gamma\right), \\
\bar{P}_{L, R}=\frac{1}{2}\left(1 \pm \gamma_{5} \Gamma_{5} \gamma_{5} / \Gamma\right),
\end{array}
$$

but these projection operators suffer from singularities in $1 / \Gamma$, as we have already noted. Namely, it is impossible to maintain the manifest CP invariance of the local and chiral symmetric doubler-free Lagrangian [12, 13, 14].

If one stays in the well-defined local Lagrangian

$$
\int \mathcal{L}_{L}=\int \bar{\psi} P_{+} D \hat{P}_{-} \psi
$$

where

$$
\begin{aligned}
& P_{ \pm}=\frac{1}{2}\left(1 \pm \gamma_{5}\right), \\
& \hat{P}_{ \pm}=\frac{1}{2}\left(1 \pm \hat{\gamma}_{5}\right)
\end{aligned}
$$

it is not invariant under the standard CP transformation as

$$
\begin{aligned}
& W P_{ \pm} W^{-1}=P_{\mp}^{T} \neq \hat{P}_{\mp}^{T}(U), \\
& W \hat{P}_{ \pm}\left(U^{\mathrm{CP}}\right) W^{-1}=\frac{1 \mp\left[\gamma_{5} \hat{\gamma}_{5}(U) \gamma_{5}\right]^{T}}{2}=\left[\gamma_{5} \hat{P}_{\mp}(U) \gamma_{5}\right]^{T} \neq P_{\mp}^{T}, \\
& {\left[W P_{+} D\left(U^{\mathrm{CP}}\right) \hat{P}_{-}\left(U^{\mathrm{CP}}\right) W^{-1}\right]^{T}=\gamma_{5} \hat{P}_{+}(U) \gamma_{5} D(U) P_{-}} \\
& =P_{+} D(U) \hat{P}_{-}(U)-D(U)\left[\gamma_{5}-\Gamma_{5}(U)\right] \neq P_{+} D \hat{P}_{-} .
\end{aligned}
$$

This generalizes the analysis of Hasenfratz in a more general setting ${ }^{3}$.

\footnotetext{
${ }^{2}$ The vector-like theory is invariant under this $\mathrm{CP}$ transformation.

${ }^{3}$ This analysis is extended to a more general class of operators defined by $\gamma_{5} H+H \gamma_{5}=2 H^{2} f\left(H^{2}\right)$, where $f\left(H^{2}\right)$ is a regular and monotonous non-decreasing function[13] .
} 


\section{Majorana fermion}

We next want to show that the general class of Ginsparg-Wilson operators have a difficulty to define Majorana fermions in the presence of chiral symmetric Yukawa couplings[10]. We start with

$$
\begin{aligned}
\mathcal{L}= & \bar{\psi}_{R} D \psi_{R}+\bar{\psi}_{L} D \psi_{L}+m\left[\bar{\psi}_{R} \psi_{L}+\bar{\psi}_{L} \psi_{R}\right] \\
& +2 g\left[\bar{\psi}_{L} \phi \psi_{R}+\bar{\psi}_{R} \phi^{\dagger} \psi_{L}\right] \\
= & \bar{\psi} D \psi+m \bar{\psi} \gamma_{5} \Gamma_{5} \psi \\
& +\frac{g}{\sqrt{2}} \bar{\psi}\left[A+\left(\gamma_{5} \Gamma_{5} \gamma_{5} / \Gamma\right) A\left(\Gamma_{5} / \Gamma\right)+i\left(\gamma_{5} \Gamma_{5} \gamma_{5} / \Gamma\right) B+i B\left(\Gamma_{5} / \Gamma\right)\right] \psi
\end{aligned}
$$

where

$$
\psi_{L, R}=P_{L, R} \psi, \quad \bar{\psi}_{L, R}=\bar{\psi} \bar{P}_{L, R}
$$

with the projection operators in $(23)$, and we used $\phi=(A+i B) / \sqrt{2}$. We then make the substitution $[15,16]$

$$
\begin{aligned}
& \psi=(\chi+i \eta) / \sqrt{2}, \\
& \bar{\psi}=\left(\chi^{T} C-i \eta^{T} C\right) / \sqrt{2}
\end{aligned}
$$

and obtain

$$
\begin{aligned}
\mathcal{L}= & \frac{1}{2} \chi^{T} C D \chi+\frac{1}{2} m \chi^{T} C \gamma_{5} \Gamma_{5} \chi \\
& +\frac{g}{2 \sqrt{2}} \chi^{T} C\left[A+\left(\gamma_{5} \Gamma_{5} \gamma_{5} / \Gamma\right) A\left(\Gamma_{5} / \Gamma\right)+i\left(\gamma_{5} \Gamma_{5} \gamma_{5} / \Gamma\right) B+i B\left(\Gamma_{5} / \Gamma\right)\right] \chi \\
+ & \frac{1}{2} \eta^{T} C D \eta+\frac{1}{2} m \eta^{T} C \gamma_{5} \Gamma_{5} \eta \\
& +\frac{g}{2 \sqrt{2}} \eta^{T} C\left[A+\left(\gamma_{5} \Gamma_{5} \gamma_{5} / \Gamma\right) A\left(\Gamma_{5} / \Gamma\right)+i\left(\gamma_{5} \Gamma_{5} \gamma_{5} / \Gamma\right) B+i B\left(\Gamma_{5} / \Gamma\right)\right] \eta
\end{aligned}
$$

This relation shows that we can write the Dirac fermion operator as a sum of two Majorana operators. One can then define the Majorana fermion $\chi$ (or $\eta$ ) and the resulting Pfaffian as a square root of the determinant of lattice Dirac operator. But this formulation of the Majorana fermion inevitably suffers from the singularities of the modified chiral operators $\Gamma_{5} / \Gamma$ and $\gamma_{5} \Gamma_{5} \gamma_{5} / \Gamma$ in the Brillouin zone, as we have already explained.

We note that the condition,

$$
C P_{L} C^{-1}=\bar{P}_{R}^{T}, \quad C \bar{P}_{L} C^{-1}=P_{R}^{T}
$$

which is required by the consistent $C P$ property, is directly related to the condition of the consistent Majorana reduction for the term containing scalar field $A(x)$,

$$
C\left(\gamma_{5} \Gamma_{5} \gamma_{5} / \Gamma\right) A(x)\left(\Gamma_{5} / \Gamma\right)=-\left[C\left(\gamma_{5} \Gamma_{5} \gamma_{5} / \Gamma\right) A(x)\left(\Gamma_{5} / \Gamma\right)\right]^{T}
$$

in the Yukawa coupling ${ }^{4}$, if one recalls that the difference operators in $\Gamma_{5}$ and $\Gamma$ do not commute with the field $A(x)$.

\footnotetext{
${ }^{4}$ If $(C O)^{T}=-C O$ for a general operator $O$, the cross term vanishes $\eta^{T} C O \chi-\chi^{T} C O \eta=0$ by using the anti-commuting property of $\chi$ and $\eta$.
} 
In other words, if one uses the projection operators which do not satisfy,

$$
C P_{L} C^{-1}=\bar{P}_{R}^{T}, \quad C \bar{P}_{L} C^{-1}=P_{R}^{T}
$$

the consistent Majorana reduction is not realized. For the chiral symmetric Yukawa couplings such as in supersymmetry the Majorana reduction is thus directly related to the condition of the CP invariance, provided that parity properties are the standard ones.

\section{Discussion and conclusion}

We have shown that both of the consistent definitions of CP symmetry in chiral gauge theory and the Majorana fermion in the presence of chiral symmetric Yukawa couplings are based on the same condition,

$$
C P_{L} C^{-1}=\bar{P}_{R}^{T}, \quad C \bar{P}_{L} C^{-1}=P_{R}^{T}
$$

and that the construction of projection operators, which are the unique solution,

$$
\begin{array}{r}
P_{L, R}=\frac{1}{2}\left(1 \mp \Gamma_{5} / \Gamma\right), \\
\bar{P}_{L, R}=\frac{1}{2}\left(1 \pm \gamma_{5} \Gamma_{5} \gamma_{5} / \Gamma\right)
\end{array}
$$

inevitably suffers from singularities in the modified chiral operators (to be precise, in $1 / \Gamma$ ) for any Dirac operator $D$ satisfying the algebraic relation

$$
\gamma_{5} H+H \gamma_{5}=2 H^{2 k+2}
$$

with $H=a \gamma_{5} D$. We find it interesting that the breaking of CP symmetry and a conflict with Majorana reduction are directly related to the basic notions of locality and species doubling in lattice theory.

If one uses well-defined regular lattice operators

$$
\int \mathcal{D} \psi_{L} \mathcal{D} \bar{\psi}_{L} \exp \left(\int \bar{\psi} P_{+} D \hat{P}_{-} \psi\right)
$$

with

$$
\begin{aligned}
& P_{ \pm}=\frac{1}{2}\left(1 \pm \gamma_{5}\right) \\
& \hat{P}_{ \pm}=\frac{1}{2}\left(1 \pm \hat{\gamma}_{5}\right)
\end{aligned}
$$

the CP breaking in lattice chiral theory on the basis of Ginsparg-Wilson operator inevitably appears. As for the physical implications of this breaking of CP symmetry in pure lattice chiral theory, it was shown in Ref.[14] following the formulation in[17, 18, 19, 20] that the effects of CP breaking are isolated in the (almost) contact term of fermion propagator, which is connected to the external fermion sources. The CP breaking effects in all 
the loop diagrams are under well-control and they are absorbed into the weight factors related to various topological sectors in the fermionic path integral. In the presence of the Higgs coupling and in particular in the presence of the vacuum expectation value of the Higgs field, the analysis of CP breaking becomes more involved and it could be more serious[14].

It is also shown that this complication of CP breaking persists in the domain-wall fermion $[21,22,23,24,25]$. In fact, it is shown that the domain-wall fermion in the limit $N=\infty$, when applied to chiral theory, is valid only for the topologically trivial sector and that it still suffers from the complications related to CP symmetry[26]. In the $N=\infty$ limit, the domain-wall fermion is related to the Ginsparg-Wilson fermion by

$$
\begin{aligned}
& \int \mathcal{D} \psi_{L} \mathcal{D} \bar{\psi}_{L} \exp \left(\int \bar{\psi} P_{+} D \hat{P}_{-} \psi\right) \\
& =\int \mathcal{D} q_{L} \mathcal{D} \bar{q}_{L} \mathcal{D} Q_{L} \mathcal{D} \bar{Q}_{R} \exp \left(\int \bar{q} P_{+} D \frac{1}{\gamma_{5} \Gamma_{5}} P_{-} q+\int \bar{Q} \hat{P}_{-} \frac{1}{\gamma_{5} \Gamma_{5}} P_{-} Q\right)
\end{aligned}
$$

where $q$ and $\bar{q}$ stand for the standard variables in domain-wall fermion, and $Q$ and $\bar{Q}$ stand for the Pauli-Villars fields. Note the appearance of the non-local operator $D /\left(\gamma_{5} \Gamma_{5}\right)$.

It is customary to use the domain-wall fermion for finite $N$ in practical applications, but we consider that the finite $N$ theory is not in a better situation with respect to CP symmetry either ${ }^{5}$, besides the ill-defined chiral symmetry.

\section{References}

[1] P.H. Ginsparg and K.G. Wilson, Phys. Rev. D 25 (1982) 2649.

[2] H. Neuberger, Phys. Lett. B 417 (1998) 141; Phys. Lett. B 427 (1998) 353.

[3] P. Hasenfratz, V. Laliena and F. Niedermayer, Phys. Lett. B 427 (1998) 125.

[4] M. Lüscher, Phys. Lett. B 428 (1998) 342.

[5] K. Fujikawa, Phys. Rev. Lett. 42 (1979) 1195; Phys. Rev. D 21 (1980) 2848; [Erratumibid. D 22 (1980) 1499].

[6] Y. Kikukawa and A. Yamada, Phys. Lett. B 448 (1999) 265;

D.H. Adams, Annals Phys. 296 (2002) 131;

H. Suzuki, Prog. Theor. Phys. 102 (1999) 141;

K. Fujikawa, Nucl. Phys. B 546 (1999) 480.

[7] F. Niedermayer, Nucl. Phys. (Proc. Suppl.) 73 (1999) 105;

H. Neuberger, Ann. Rev. Nucl. Part. Sci. 51 (2001) 23;

\footnotetext{
${ }^{5}$ One may argue that the domain wall variables $q_{L}$ and $\bar{q}_{L}$, which become non-local and cannot describe topological properties in the limit $N=\infty$, are not the suitable variables to describe physical correlation functions even for finite $N$, to the extent that the finite $N$ theory is intended to be an approximation to the theory with $N=\infty$.
} 
M. Lüscher, lectures given at the International School of Subnuclear physics, Erice 2000, hep-th/0102028;

Y. Kikukawa, Nucl. Phys. (Proc. Suppl.) 106 (2002) 71.

[8] K. Fujikawa, Nucl. Phys. B 589 (2000) 487.

[9] K. Fujikawa and M. Ishibashi, Nucl. Phys. B 587 (2000) 419; Nucl. Phys. B 605 (2001) 365.

[10] K. Fujikawa and M. Ishibashi, Nucl. Phys. B 622 (2002) 115; Phys. Lett. B 528 (2002) 295.

[11] T.W. Chiu, C.W. Wang and S.V. Zenkin, Phys. Lett. B 438 (1998) 321.

[12] P. Hasenfratz, Nucl. Phys. (Proc. Suppl.) 106 (2002) 159, and references therein.

[13] K. Fujikawa, M. Ishibashi and H. Suzuki, Phys. Lett. B 538 (2002) 197.

[14] K. Fujiwawa, M. Ishibashi and H. Suzuki, J. High Energy Phys. 04 (2002) 046.

[15] H. Nicolai, Nucl. Phys. B 140 (1978) 294; Nucl. Phys. B 156 (1979) 157.

[16] P. van Nieuwenhuizen and A. Waldron, Phys. Lett. B 389 (1996) 29.

[17] M. Lüscher, Nucl. Phys. B 549 (1999) 295.

[18] H. Suzuki, Prog. Theor. Phys. 101 (1999) 1147.

[19] M. Lüscher, Nucl. Phys. B 568 (2000) 162.

[20] H. Suzuki, Nucl. Phys. B 585 (2000) 471;

H. Igarashi, K. Okuyama and H. Suzuki, hep-lat/0012018.

[21] D.B. Kaplan, Phys. Lett. B 288 (1992) 342.

[22] Y. Shamir, Nucl. Phys. B 406 (1993) 90;

V. Furman and Y. Shamir, Nucl. Phys. B 439 (1995) 54.

[23] P. Vranas, Phys. Rev. D 57 (1998) 1415.

[24] H. Neuberger, Phys. Rev. D 57 (1998) 5417; Phys. Rev. D 59 (1999) 085006.

[25] Y. Kikukawa and T. Noguchi, hep-lat/9902022.

[26] K. Fujikawa and H, Suzuki, hep-lat/0210013 (To appear in Phys. Rev. D). 